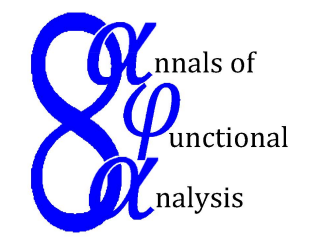

Ann. Funct. Anal. 5 (2014), no. 1, 70-76

$\mathscr{A}$ NNALS OF $\mathscr{F}$ UNCTIONAL $\mathscr{A}$ NALYSIS

ISSN: 2008-8752 (electronic)

URL:www.emis.de/journals/AFA/

\title{
ON REVERSING OF THE MODIFIED YOUNG INEQUALITY
}

\author{
A. SALEMI AND A. SHEIKH HOSSEINI* \\ This paper is dedicated to Professor Tsuyoshi Ando
}

Communicated by J. I. Fujii

\begin{abstract}
In the present paper, by Haagerup theorem, we show that if $A \in \mathbb{M}_{n}$ is a non scalar strictly positive matrix and $0<\nu<1$ be a real number such that $\nu \neq \frac{1}{2}$, then there exists $X \in \mathbb{M}_{n}$ such that
\end{abstract}

$$
\left\|A^{\nu} X A^{1-\nu}\right\|>\|\nu A X+(1-\nu) X A\|
$$

\section{INTRODUCTION AND PRELIMINARIES}

Let $\mathbb{M}_{n}$ be the algebra of all $n \times n$ complex matrices. A norm $\|\mid\| \cdot \|$ on $\mathbb{M}_{n}$ is said to be unitarily invariant if $\|U A V\|\|=\| A\|\|$ for all $A \in \mathbb{M}_{n}$ and all unitary $U, V \in \mathbb{M}_{n}$. For $A \in \mathbb{M}_{n}$, the numerical radius of $A$ is defined and denoted by

$$
\omega(A)=\max \left\{\left|x^{*} A x\right|: x \in \mathbb{C}^{n}, x^{*} x=1\right\} .
$$

It is known that $\omega($.$) is a vector norm on \mathbb{M}_{n}$, but is not unitarily invariant.

Throughout the paper we use the term positive for a positive semidefinite matrix, and strictly positive for a positive definite matrix. Also we use the notation $A \geq 0$ to mean that $A$ is positive, $A>0$ to mean it is strictly positive. In $\mathbb{M}_{n}$, beside the usual matrix product, the entrywise product is quite important and interesting. The entry wise product of two matrices $A$ and $B$ is called their Schur (or Hadamard) product and denoted by $A \circ B$. With this multiplication $\mathbb{M}_{n}$ becomes a commutative algebra, for which the matrix with all entries equal to one is the unit and we denote that by " $J$ ". The linear operator $S_{A}$ on $\mathbb{M}_{n}$, is

Date: Received: 12 June 2013; Revised: 1 August 2013; Accepted: 2 September 2013.

* Corresponding author.

2010 Mathematics Subject Classification. Primary 15A60; Secondary 15A42, 47A30.

Key words and phrases. Young inequality, numerical radius, spectral norm, strictly positive matrix. 
called the Schur multiplier operator and defined by $S_{A}(X):=A \circ X$. The induced norm of $S_{A}$ with respect to all unitarily invariant norm will be denoted by

$$
\left\|S_{A}\right\|=\sup _{X \neq 0} \frac{\left\|S_{A}(X)\right\| \|}{\|X \mid\|}=\sup _{X \neq 0} \frac{\|A \circ X\|}{\|X\|},
$$

and the induced norm of $S_{A}$ with respect to numerical radius norm will be denoted by

$$
\left\|S_{A}\right\|_{\omega}=\sup _{X \neq 0} \frac{\omega\left(S_{A}(X)\right)}{\omega(X)}=\sup _{X \neq 0} \frac{\omega(A \circ X)}{\omega(X)} .
$$

For positive real numbers $a, b$, the classical Young inequality says that if $p, q>1$ such that $1 / p+1 / q=1$, then

$$
a b \leq \frac{a^{p}}{p}+\frac{b^{q}}{q}
$$

the another form of the inequality for positive real numbers $a, b$ is in the following form:

$$
a^{\nu} b^{1-\nu} \leq \nu a+(1-\nu) b, 0 \leq \nu \leq 1
$$

For more details about these inequalities, their refinements and associated norm inequalities with their history of origin, the reader may refer to [2, 5, 6, 8, 9]. In [9] we showed that, if $A, B \geq 0$, and $X \in \mathbb{M}_{n}$. Then the inequality $\omega(A X B) \leq \omega\left(\frac{A^{p}}{p} X+X \frac{B^{q}}{q}\right)$ does not holds in general as follows:

Theorem 1.1. [9, Theorem 2.3], Let $p>q>1$ such that $1 / p+1 / q=1$ and let $A \in \mathbb{M}_{n}$ be a non scalar strictly positive matrix such that $1 \in \sigma(A)$, then there exists $X \in \mathbb{M}_{n}$ such that

$$
\omega(A X A)>\omega\left(\frac{A^{p}}{p} X+X \frac{A^{q}}{q}\right) .
$$

Also, in [10] we showed the following inequaliy for the numerical radius:

Theorem 1.2. Let $A \in \mathbb{M}_{n}$ be a positive matrix. Then for all $X \in \mathbb{M}_{n}$, we have

$$
\omega(A X A) \leq \frac{1}{2} \omega\left(A^{2} X+X A^{2}\right)
$$

\section{MAin RESUlts}

Bhatia and Kittaneh in 1990 [7] established a matrix mean inequality as follows:

$$
\left\|A^{*} B\right\| \mid \leq \frac{1}{2}\left\|A^{*} A+B^{*} B\right\| \|
$$

for matrices $A, B \in \mathbb{M}_{n}$.

In [5] a generalization of (2.1) was proved, for all $X \in \mathbb{M}_{n}$,

$$
\left\|A^{*} X B\right\| \mid \leq \frac{1}{2}\left\|A A^{*} X+X B B^{*}\right\| \| .
$$


Ando in 1995 [2] obtained a matrix Young inequality:

$$
\|A B\| \leq\|\| \frac{A^{p}}{p}+\frac{B^{q}}{q}\|\|,
$$

for $p, q>1$ with $1 / p+1 / q=1$ and positive matrices $A, B$.

Also, in [1], the author pointed out that the matrix Young inequality $\|A X B\|\|\leq\|\left|\frac{1}{p} A^{p} X+\frac{1}{q} X B^{q} \|\right| \mid$ is not valid for the spectral norm $\|$.$\| .$

Here, we clarify it. Ando and Okubo in 1991, [4], proved the following theorem[4, Theorom 1 and Corollary 3]:

Theorem 2.1. (Haagerup theorem) For $A \in \mathbb{M}_{n}$ the following assertions are equivalent:

(i) $\left\|S_{A}\right\| \leq 1$.

(ii) There is $0 \leq R_{1}, R_{2} \in \mathbb{M}_{n}$ such that

$$
\left[\begin{array}{cc}
R_{1} & A \\
A^{*} & R_{2}
\end{array}\right] \geq 0, \quad R_{1} \circ I \leq I \quad \text { and } \quad R_{2} \circ I \leq I .
$$

Moreover, if $A$ is Hermitian, then $\left\|S_{A}\right\|=\left\|S_{A}\right\|_{\omega}$.

Lemma 2.2. Let $0<\nu<1$ be a real number such that $\nu \neq \frac{1}{2}$ and $a>0$. Then

$$
\frac{a^{\nu}}{\nu a+(1-\nu)}=\frac{a^{1-\nu}}{\nu+(1-\nu) a}
$$

holds if and only if $a=1$.

Proof. Assume if possible there exists $a>0$ and $a \neq 1$, such that

$$
\frac{a^{\nu}}{\nu a+(1-\nu)}=\frac{a^{1-\nu}}{\nu+(1-\nu) a} \text {. }
$$

Then (2.4) is equivalent to

$$
(1-\nu) a^{1+\nu}+\nu a^{\nu}-\nu a^{2-\nu}-(1-\nu) a^{1-\nu}=0 .
$$

Now let $\nu=\frac{1}{p}$, where $p>1, p \neq 2$ and let

$$
f(x)=\frac{1}{p}\left((p-1) x^{\frac{p+1}{p}}+x^{\frac{1}{p}}-x^{\frac{2 p-1}{p}}-(p-1) x^{\frac{p-1}{p}}\right) .
$$

Now replace $x$ with $x^{p}$ we have

$$
k(x)=\frac{1}{p}\left((p-1) x^{p+1}+x-x^{2 p-1}-(p-1) x^{p-1}\right)=\frac{x k_{1}(x)}{p} .
$$

By the assumption and by the Rolle's theorem, the (2.5) is equivalent to

$$
k_{1}(x)=(p-1) x^{p}-x^{2 p-2}-(p-1) x^{p-2}+1
$$

has at least one positive root $r_{1} \neq 1$. Now, apply the Rolle's theorem for

$$
k_{2}(x)=k_{1}^{\prime}(x)=(p-1) x^{p-3}\left(p x^{2}-2 x^{p}-(p-2)\right)=(p-1) x^{p-3} k_{3}(x),
$$

we can say that the function

$$
k_{3}^{\prime}(x)=2 p x\left(1-x^{p-2}\right)
$$


has at least one positive root $r_{2} \neq 1$. That is a contradiction.

Now, in the following theorem, we will show that if $A, B \geq 0$, and $X \in \mathbb{M}_{n}$, then $\left\|A^{\nu} X B^{1-\nu}\right\| \leq\|\| \nu A X+(1-\nu) X B\|\|$ does not holds in general.

Theorem 2.3. Let $0<\nu<1$ be a real number such that $\nu \neq \frac{1}{2}$ and $A \in \mathbb{M}_{n}$ be a non scalar strictly positive matrix. Then there exists $X \in \mathbb{M}_{n}^{2}$ such that

$$
\left\|A^{\nu} X A^{1-\nu}\right\|>\|\nu A X+(1-\nu) X A\| \text {. }
$$

Proof. Without loss of generality we assume that $A=\operatorname{diag}\left(a_{1}, a_{2}, a_{3}, \ldots, a_{n}\right)$ where $a_{1}=1$ and $a_{2} \neq 1$. By Lemma 2.2 , it is readily seen that

$$
\frac{a_{2}^{\nu}}{\nu a_{2}+(1-\nu)} \neq \frac{a_{2}^{1-\nu}}{\nu+(1-\nu) a_{2}} \text {. }
$$

Assume if possible for all $X \in \mathbb{M}_{n}$,

$$
\left\|A^{\nu} X A^{1-\nu}\right\| \leq\|\nu A X+(1-\nu) X A\| .
$$

Now, let $C=\left(c_{i j}\right)$ and $E=\left(e_{i j}\right)$ be $n \times n$ matrices, where $c_{i j}=\nu a_{i}+(1-\nu) a_{j}$, and $e_{i j}=a_{i}^{\nu} a_{j}^{1-\nu}$. Then we rewrite (2.8) in the following form

$$
\|E \circ X\| \leq\|C \circ X\|, \quad\left(X \in \mathbb{M}_{n}\right) .
$$

Let $D$ be the entrywise inverse of $C(C \circ D=J)$. We replace $X$ by $(D \circ X)$ in (2.9), then

$$
\|(E \circ D) \circ X\| \leq\|X\|, \quad\left(X \in \mathbb{M}_{n}\right) .
$$

Let $F:=(E \circ D)=\left(f_{i j}\right)$. Then $\|F \circ X\| \leq\|X\|$ for all $X \in \mathbb{M}_{n}$ and hence,

$$
\left\|S_{F}\right\| \leq 1
$$

Now by Haagerup theorem, there exist $n \times n$ matrices $X=\left(x_{i j}\right), Y=\left(y_{i j}\right) \geq 0$ with $0 \leq x_{i i}, y_{i i} \leq 1, \quad(1 \leq i \leq n)$, such that

$$
\left[\begin{array}{ll}
X & F \\
F^{*} & Y
\end{array}\right] \geq 0
$$

By considering $\tilde{X}:=\left(\tilde{x}_{i j}\right)$ such that $\tilde{x}_{i j}=x_{i j}$ if $i \neq j$ and $\tilde{x}_{i i}=1$, and $\tilde{Y}:=\left(\tilde{y}_{i j}\right)$ such that $\tilde{y}_{i j}=y_{i j}$ if $i \neq j$ and $\tilde{y}_{i i}=1$, we obtain that

$$
\left[\begin{array}{ll}
\tilde{X} & F \\
F^{*} & \tilde{Y}
\end{array}\right] \geq\left[\begin{array}{ll}
X & F \\
F^{*} & Y
\end{array}\right] \geq 0
$$

Since, any principal submatrix of the above matrix is positive, we have

$$
\left[\begin{array}{cccc}
1 & x & 1 & f_{12} \\
\bar{x} & 1 & f_{21} & 1 \\
1 & f_{21} & 1 & y \\
f_{12} & 1 & \bar{y} & 1
\end{array}\right] \geq 0 \text { where } x:=\tilde{x}_{12}=x_{12}, y:=\tilde{y}_{12}=y_{12} .
$$

By using the Schur complement Theorem [5, Theorem 1.3.3], we obtain that

$$
\left[\begin{array}{ccc}
1 & f_{21} & 1 \\
f_{21} & 1 & y \\
1 & \bar{y} & 1
\end{array}\right]-\left[\begin{array}{c}
\bar{x} \\
1 \\
f_{12}
\end{array}\right]\left[\begin{array}{lll}
x & 1 & f_{12}
\end{array}\right]=\left[\begin{array}{ccc}
1-|x|^{2} & f_{21}-\bar{x} & 1-\bar{x} f_{12} \\
f_{21}-x & 0 & y-f_{12} \\
1-x f_{12} & \bar{y}-f_{12} & 1-f_{12}^{2}
\end{array}\right] \geq 0 .
$$


Since the determinant of principle submatrices of the above matrix is positive, we have $f_{21}-x=y-f_{12}=0$ and hence

$$
B=\left[\begin{array}{cccc}
1 & f_{21} & 1 & f_{12} \\
f_{21} & 1 & f_{21} & 1 \\
1 & f_{21} & 1 & f_{12} \\
f_{12} & 1 & f_{12} & 1
\end{array}\right] \geq 0
$$

Let $f(\lambda)$ be the characteristic polynomial of $B$ as follows

$$
f(\lambda)=\lambda^{4}-4 \lambda^{3}+\left(4-2 f_{12}^{2}-2 f_{21}^{2}\right) \lambda^{2}+\left(-4 f_{12} f_{21}+2 f_{12}^{2}+2 f_{21}^{2}\right) \lambda .
$$

By (2.7) we have $f_{21} \neq f_{12}$, we obtain that the coefficient of $\lambda$ is positive and hence $f(\lambda)$ has one negative root, which is a contradiction with $B \geq 0$.

Corollary 2.4. Let $p>q>1$ such that $1 / p+1 / q=1$ and $n \in \mathbb{N}$. Then there exist $A, B, X \in \mathbb{M}_{n}$ such that $A, B>0$ and

$$
\|A X B\|>\left\|\frac{A^{p}}{p} X+X \frac{B^{q}}{q}\right\| .
$$

Lemma 2.5. [4] For all $A \in \mathbb{M}_{n}$

$$
\left\|S_{A}\right\| \leq\left\|S_{A}\right\|_{\omega}
$$

Now, by Lemma 2.5 and Theorem 2.3 we can obtain the following theorem that shows the another form of the Young inequality for the numerical radius does not holds.

Theorem 2.6. Let $0<\nu<1$ be a real number such that $\nu \neq \frac{1}{2}$ and $A \in \mathbb{M}_{n}$ be a non scalar strictly positive matrix. Then there exists $X \in \mathbb{M}_{n}^{2}$ such that

$$
\omega\left(A^{\nu} X A^{1-\nu}\right)>\omega(\nu A X+(1-\nu) X A) .
$$

Proof. Without loss of generality we assume that $A=\operatorname{diag}\left(a_{1}, a_{2}, a_{3}, \ldots, a_{n}\right)$. We assume if possible for all $A, X \in \mathbb{M}_{n}$ such that $A$ is a non scalar strictly positive matrix, then

If we define

$$
\omega\left(A^{\nu} X A^{1-\nu}\right) \leq \omega(\nu A X+(1-\nu) X A)
$$

$$
F:=\left[\frac{a_{i}^{\nu} a_{j}^{1-\nu}}{\nu a_{i}+(1-\nu) a_{j}}\right] \in \mathbb{M}_{n},
$$

then easy computations show that $\left\|S_{F}\right\|_{\omega} \leq 1$. Now by Lemma 2.5 we have $\left\|S_{F}\right\| \leq 1$ and hence $\left\|A^{\nu} X A^{1-\nu}\right\| \leq\|\nu A X+(1-\nu) X A\|$, which is a contradiction by Theorem 2.3 .

Corollary 2.7. Let $p>q>1$ such that $1 / p+1 / q=1$ and $n \in \mathbb{N}$. Then there exist $A, B, X \in \mathbb{M}_{n}$ such that $A, B>0$ and

$$
\omega(A X B)>\omega\left(\frac{A^{p}}{p} X+X \frac{B^{q}}{q}\right) .
$$

Remark 2.8. By the inequality (2.2) and Theorem 1.2, the condition $\nu \neq \frac{1}{2}$ in the Theorem 2.3 and Theorem 2.6 are essential. 
Theorem 2.9. Let $p>q>1$ such that $1 / p+1 / q=1$. Then there is $A \in \mathbb{M}_{n}$ such that $A>0$ and for all $X \in \mathbb{M}_{n}$

$$
\|A X A\| \leq\|\| \frac{A^{p}}{p} X+X \frac{A^{q}}{q}\|\|
$$

if and only if there is

$$
F=\left[\frac{a_{i} a_{j}}{\frac{a_{i}^{p}}{p}+\frac{a_{j}^{q}}{q}}\right] \in \mathbb{M}_{n},
$$

such that $a_{i}>0(i=1, \ldots, n)$ and $\left\|\mid S_{F}\right\| \leq 1$.

Moreover, if $A$ is non scalar and $1 \in \sigma(A)$, then $\left\|S_{F}\right\|_{\omega}>\left\|S_{F}\right\|$.

Proof. Without loss of generality, assume that

$$
A=\operatorname{diag}\left(a_{1}, a_{2}, a_{3}, \ldots, a_{n}\right), \quad a_{i}>0,(i=1, \ldots, n)
$$

Now, let $C=\left[c_{i j}\right]$ and $E=\left[e_{i j}\right]$ be $n \times n$ matrices, where

$$
c_{i j}=\frac{a_{i}^{p}}{p}+\frac{a_{j}^{q}}{q}, \quad e_{i j}=a_{i} a_{j} .
$$

Then we have the following form

$$
\|E \circ X\| \mid \leq\|C \circ X\| \|, \quad\left(X \in \mathbb{M}_{n}\right) .
$$

Let $D$ be the entrywise inverse of $C(C \circ D=J)$. We replace $X$ by $(D \circ X)$ in (2.12), then

$$
\|(E \circ D) \circ X|\|\leq\| X|\|, \quad\left(X \in \mathbb{M}_{n}\right) .
$$

Let $F:=(E \circ D)=\left(f_{i j}\right)$. Then, we obtain that

$$
\|F \circ X\| \leq\|X\|, \quad\left(X \in \mathbb{M}_{n}\right)
$$

and hence, $\left|\left\|S_{F} \mid\right\| \leq 1\right.$. It is enough to show that if $A$ is non scalar and $1 \in \sigma(A)$, then $\left\|S_{F}\right\|_{\omega}>1$. Assume if possible $\left\|S_{F}\right\|_{\omega} \leq 1$. Then we have for all $X \in \mathbb{M}_{n}$,

$$
\omega(A X A) \leq \omega\left(\frac{A^{p}}{p} X+X \frac{A^{q}}{q}\right) .
$$

That is a contradiction by Theorem 1.1.

\section{REFERENCES}

1. T. Ando, Majorization and inequalities in matrix theory, Linear Algebra Appl. 199 (1994), $17-67$.

2. T. Ando, Matrix Young inequalities, Oper. Theory Adv. Appl. 75 (1995), 33-38.

3. T. Ando, R.A. Horn and C.R. Johnson, The singular values of a Hadamard product: A basic inequality, Linear Multilinear Algebra 21 (1987), 345-365.

4. T. Ando and K. Okubo, Induced norms of the Schur multiplication operators, Linear Algebra Appl. 147(1991), 181-199.

5. R. Bhatia, Positive Definite Matrices, Princeton University Press, 2007.

6. R. Bhatia and C. Davis, More matrix forms of the arithmetic-geometric mean inequality, SIAM J. Matrix Anal. Appl. 14(1993), 132-136.

7. R. Bhatia and F. Kittaneh, On the singular values of a product of operators, SIAM J. Matrix Anal. Appl. 11 (1990), 272-277. 
8. C. Conde, Young type inequalities for positive operators, Ann. Funct. Anal. 2 (2013), no. $2,144-152$.

9. A. Salemi and A. Sheikh Hosseini, Matrix Young numerical radius inequalities, Math. Inequal. Appl. 16 (2013), no. 3, 783-791.

10. A. Salemi and A. Sheikh Hosseini, Numerical radius and operator norm inequalities, preprint.

11. X. Zhan, Inequalities for unitarily invariant norms, SIAM J. Matrix Anal. Appl. 20 (1998) $466-470$.

12. X. Zhan, Matrix Inequalities(Lecture notes in mathematics), 1790, Springer, 2002.

Department of Mathematics, Shahid Bahonar University, Kerman, Iran.

E-mail address: salemi@mail.uk.ac.ir

E-mail address: alemehsheikhhoseiny@yahoo.com 